\begin{tabular}{|c|c|c|}
\hline \multirow{3}{*}{$\begin{array}{l}\text { ITC } \mathbf{1 / 4 8} \\
\text { Journal of Information Technology } \\
\text { and Control } \\
\text { Vol. } 48 \text { / No.1/ } 2019 \\
\text { pp. 160-171 } \\
\text { DOI 10.5755/j01.itc.48.1.20296 }\end{array}$} & \multicolumn{2}{|c|}{$\begin{array}{l}\text { Optimization of PID Controller Based on Water Wave } \\
\text { Optimization for an Automatic Voltage Regulator System }\end{array}$} \\
\hline & Received 2018/03/06 & Accepted after revision 2018/12/18 \\
\hline & \multicolumn{2}{|c|}{ Crossef http://dx.doi.org/10.5755/j01.itc.48.1.20296 } \\
\hline
\end{tabular}

\title{
Optimization of PID Controller Based on Water Wave Optimization for an Automatic Voltage Regulator System
}

\section{Yongquan Zhou}

College of Information Science and Engineering, Guangxi University for Nationalities, Nanning 530006, China Key Laboratory of Guangxi High Schools Complex System and Computational Intelligence, Nanning 530006, China; e-mail:yongquanzhou@126.com

\section{Jinzhong Zhang, Xiao Yang, Ying Ling}

College of Information Science and Engineering, Guangxi University for Nationalities, Nanning 530006, China; e-mails: zjz1436057874@163.com, yangxiaoxiaovip@126.com,yinglingly@163.com

Corresponding author: yongquanzhou@126.com

In this paper, a water wave optimization (WWO) algorithm is applied to optimize the optimal Proportional Integral Derivate (PID) controller of an Automatic Voltage Regulator (AVR) system to obtain optimal controller parameters. The WWO algorithm with good global search and local search abilities can effectively find the optimal fitness value of the problem. To verity the effectiveness of the WWO algorithm, we compare the optimization results between the WWO algorithm and other algorithms, such as the bat algorithm (BA), the crow search algorithm (CSA), the flower pollination algorithm (FPA), the particle swarm optimization (PSO) and the sine cosine algorithm (SCA). The WWO algorithm can find the optimal controller parameters and global optimal solutions by propagation operation, breaking operation and refraction operation. The calculation accuracy of the WWO algorithm is better than that of other algorithms. The experimental results show that the WWO algorithm is more efficient and robust in improving the step response of an AVR system, and the optimized results obtained by the WWO algorithm are better than other algorithms.

KEYWORDS: Water wave optimization algorithm, proportional integral derivate controller, automatic voltage regulator system, optimal controller parameters, global optimal solution. 


\section{Introduction}

The PID controller is one of the earliest proposed feedback controllers, which has the advantages of simple principle, strong robustness and high reliability, and it is widely used in industrial control systems. The traditional tuning methods, such as stabilization boundary method, decay curve method, dynamic characteristic method and Ziegler Nichols method, have been accepted to design PID controller for AVR system. To optimize the PID controller parameters to obtain the best control accuracy, some heuristic optimization algorithms have been put forward by some scholars to solve the problem, which has a wider range of applications. The genetic algorithm is used to tune the PID control gains [15]. The genetic algorithm is applied to design the self-tuning PID controllers [13]. The particle swarm optimization is presented to approach for optimal control parameters of PID in AVR system [3]. Gozde and Taplamacioglu [4] have comparatived performance analysis of artificial bee colony algorithm for AVR system. Panda, Sahu and Mohanty [16] have designed and performanced analysis of PID controller for an AVR system using simplified particle swarm optimization (PSO). Hasanien [5] has designed optimization of PID controller in AVR system using taguchi combined genetic algorithm (GA) method. The piecewise function-based gravitational search algorithm was put forward and it was applied to the parameters identification of the AVR system [11]. Tang, Zhao and Han, et al. [19] have presented the imperialist competitive algorithm for optimizing the gray PID controller of the AVR system. The teaching learning-based optimization (TLBO) algorithm optimizes PID controller for the AVR system to find out the optimal controller gains [2]. The African Buffalo Optimization (ABO) algorithm was applied to tune the PID controller parameters for an effective AVR system [14].

Optimization is an important research field, and solving optimization problems is a challenging issue. Many traditional methods are not adequate to solve complex optimization problems. During the recent years, there are many famous meta-heuristic algorithms which include bat algorithm (BA) [23], crow search algorithm (CSA) [1], flower pollination algorithm (FPA) [24], particle swarm optimization (PSO) [7], sine cosine algorithm (SCA) [12] and Water wave optimization (WWO). The WWO algorithm based on shallow wave theory was proposed by Zheng in 2015, and was applied to solve the function optimization and the high-speed rail scheduling problem [28]. Wu, et al. [20] have proposed the elite opposition-based WWO algorithm to solve function optimization and structural engineering design examples, which verified the effectiveness and feasibility of the modified algorithm. The WWO algorithm was applied to solve the economic emission dispatch problems. The results show that WWO algorithm has better overall optimization performance [17]. The combination of the water wave optimization and firefly algorithm was successfully applied to solve the IEEE-30 bus system can obtain the optimal control parameters and less active power loss [10]. Moataz Kilany, et al. [8] have introduced an approach of classifying accelerometer data for simple human activities using Support Vector Machine (SVM). Classifier results are being optimized by WWO Algorithm. Yun et al. [25] have used the WWO algorithm for flow-shop scheduling problem, and the overall optimization performance of WWO was superior to other algorithms. The WWO algorithm was used to solve the digital IIR filter design problem, and obtained the better results [27]. The WWO algorithm was applied to solve the multi-area economic dispatch problem, and found the global optimal solution [9]. The WWO algorithm was applied to solve the combined economic and emission dispatch problem, and the results shown that the WWO algorithm had certain advantages in solving this problem [18]. Wu et al. [21] have proposed the water wave optimization algorithm to solve the traveling salesman problem. The WWO algorithm was better than other algorithms. The WWO algorithm was applied to image match and achieved a better match [22]. The combination of the min-max technique and water wave optimization algorithm was applied to solve the multi-objective bidding strategy problem [6]. Zhang et al. [26] have proposed an improved sine cosine water wave optimization algorithm to solve the function optimization problem, and obtained the faster convergence speed and higher calculation accuracy.

The WWO algorithm is applied to optimize the PID controller parameters for an AVR system. The aim of optimization is to determine the parameters of the PID controller, so that the fitness value of the objective function is optimal. Compared with other algorithms, the calculation accuracy of the WWO algorithm is much better. Therefore, the WWO algo- 
rithm has good effectiveness in the PID control parameters tuning.

The article has the following sections: Section 2 introduces an AVR system modeling; Section 3 reviews the WWO algorithm; Section 4 mainly PID controller based on the WWO algorithm are proposed; the experimental results and analysis are presented in Section 5. Finally, Section 6 discusses the conclusion and future work.

\section{Modeling of an AVR System}

Excitation control of synchronous alternator is very important for improving the power system stability and power quality. The purpose of the AVR system is to maintain the generator terminal voltage at the desired value. A simple AVR system consists of four parts: amplifier, exciter, generator and sensor. The four components must be linear, which takes into account time constant and ignores the other nonlinearity. The reasonable transfer function of these components can be given as:

\subsection{Amplifier Model}

The amplifier model is represented by a gain $K_{A}$ and a time constant $\tau_{A}$. The transfer function is given by:

$$
G_{A}(S)=\frac{K_{A}}{1+\tau_{A} s},
$$

where the range of value for $K_{A}$ is $[10,400]$, the time constant $\tau_{A}$ is very small, its value is from 0.02 to $0.1 \mathrm{~s}$.

\subsection{Exciter Model}

The transfer function of an exciter is modeled by a gain of $K_{E}$ and a time constant of $\tau_{E}$. The transfer function is represented as:

$$
G_{E}(S)=\frac{K_{E}}{1+\tau_{E} S},
$$

where $K_{E}$ and $\tau_{E}$ are constants, representing a gain and a time constant, respectively. $K_{E}$ is in the range of $[10,400]$, and $\tau_{E}$ is in the range of 0.5 to $1.0 \mathrm{~s}$.

\subsection{Generator Model}

The generator model can be represented by a gain $K_{G}$ and a time constant $\tau_{G}$, the transfer function is given by:

$$
G_{G}(S)=\frac{K_{G}}{1+\tau_{G} s},
$$

where a gain $K_{G}$ can vary from 0.7 to 1.0 , and $\tau_{G}$ varies from 1.0 to $2.0 \mathrm{~s}$.

\subsection{Sensor Model}

The sensor model can be represented by a gain $K_{S}$ and a time constant $\tau_{S}$, the transfer function is given by:

$$
G_{S}(S)=\frac{K_{S}}{1+\tau_{S} S},
$$

where a gain $K_{S}$ can vary from 10 to 400 , and $\tau_{S}$ varies from 0.001 to $0.06 \mathrm{~s}$.

The important parameters of the AVR system are taken as: $K_{A}=10, \tau_{A}=0.1, K_{E}=1.0, \tau_{E}=0.4, K_{G}=1.0$, $\tau_{G}=1.0, K_{S}=1.0$ and $\tau_{S}=0.01$. The transfer function of the AVR system can be given by:

$$
\frac{\Delta V_{t}(s)}{\Delta V_{\text {ref }}(s)}=\frac{0.1 s+10}{0.0004 s^{4}+0.0454 s^{3}+0.555 s^{2}+1.51 s+11} .
$$

The AVR system has a rise time of $0.261 \mathrm{~s}$, a settling time of $6.97 \mathrm{~s}$, steady state value of 0.909 , and peak amplitude of 1.5 volts.

\section{WWO Algorithm}

The WWO algorithm is a novel evolutionary algorithm, which mainly simulates the propagation, refraction and breaking operations to find the global optimal solution. For each water wave, it has two im-

\section{Figure 1}

Different wave shapes in deep and shallow water

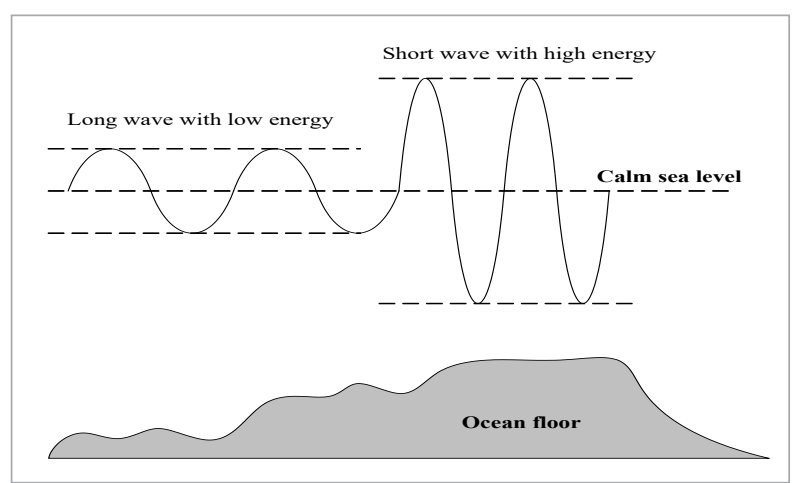


portant properties: wave height $h$ and wave length $\lambda$. They have a close relationship with the fitness value. When a wave travels from deep water (low fitness location) to shallow water (high fitness location), its wave height increases and its wavelength decreases, as illustrated in Figure 1. The correspondence between problem space and population space is shown in Table 1.

Table 1

Correspondence between problem space and population space

\begin{tabular}{l|l}
\hline Problem space & Population space \\
\hline The search space & Seabed area \\
\hline $\begin{array}{l}\text { Each solution to the } \\
\text { problem }\end{array}$ & A water wave object \\
$\begin{array}{l}\text { The fitness value of each } \\
\text { solution }\end{array}$ & $\begin{array}{l}\text { It is inversely proportional to } \\
\text { vertical distance to the seabed }\end{array}$ \\
\hline
\end{tabular}

\subsection{Propagation}

At each generation, each wave needs to be propagated exactly once. The propagation operator creates a new wave $x^{\prime}$ by shifting each dimension $d$ of the original wave $x$ as

$$
x^{\prime}(d)=x(d)+\operatorname{rand}(-1,1) \cdot \lambda L(d)
$$

where $\operatorname{rand}(-1,1)$ is a uniformly distributed random number in the range $[-1,1], L(d)$ is the length of $d$ dimension search space. If the new position is outside the feasible range, it will be reset to a random position in the range.

When a wave travels from deep water (low fitness location) to shallow water (high fitness location), its wave height increases and its wave length decreases, as illustrated in Figure 1. Thus, after propagation we calculate the fitness of the offspring wave $x^{\prime}$.

If $f\left(x^{\prime}\right)>f(x), x$ is replaced by $x^{\prime}$ in the population, and the wave height of $x^{\prime}$ is reset to $h_{\max }$. Otherwise, $x$ is remained, but its height $h$ is decreased by one, which mimics energy dissipation due to inertial resistance, vortex shedding, and bottom friction.

After each generation, the wave length of each wave $x$ is updated as follows:

$$
\lambda=\lambda \cdot \alpha^{-\left(f(x)-f_{\min }+\varepsilon\right) /\left(f_{\max }-f_{\min }+\varepsilon\right),}
$$

where $\alpha$ is the wave length attenuation coefficient of every water wave, $f_{\min }$ and $f_{\max }$ are minimum and max- imum fitness values in the entire search space, $\varepsilon$ is an infinitesimal positive integer to avoid division-by-zero.

\subsection{Breaking}

In WWO algorithm, the velocity of the crest exceeds the velocity of the water wave, and the energy of the water wave is increasing. At the same time, the water wave becomes steeper and steeper, and the water wave will break into a series of solitary waves. Once the optimal water wave $x^{*}$ is searched, it will be executed by breaking operation. In detail, we randomly select $k$ dimensions (where $k$ is a random number between 1 and $k_{\max }$ ), and at each dimension $d$ generate a solitary wave $x^{\prime}$. The location update can be given as:

$$
x^{\prime}(d)=x(d)+N(0,1) \cdot \beta L(d),
$$

where $\beta$ is the broken wave coefficient and its range is 0.001 to 0.01 . If none of the solitary waves are better than $x^{*}, x^{*}$ is remained; Otherwise $x^{*}$ is replaced by the fitness one among the solitary waves.

\subsection{Refraction}

In wave propagation, if flowing through steep places with deep sea features like valleys and mountain, the water wave is not perpendicular to the isobaths, its direction is deflected. The water waves converge in shallow waters and diverge in deep water areas. In order to better simulate the losses of water wave energy, we will reduce the wave height $h$ by one. After multiple propagation operations, the wave height $h$ is reduced to zero, and use a simple way to calculate the position after refraction.

$$
x^{\prime}(d)=N\left(\frac{(x *(d)+x(d))}{2}, \frac{|x *(d)-x(d)|}{2}\right),
$$

where $x^{*}$ is the best solution in the current population, $N(\mu, \sigma)$ is a Gaussian random number with mean $\mu$ and standard deviation $\sigma$. The new position is between the original position and the best known position, and the standard deviation $\sigma$ is equal to the absolute value of their difference. After the refraction operation, the wave height is set to $h_{\max }$, and at the same time, the wavelength is updated as follows:

$$
\lambda^{\prime}=\lambda \frac{f(x)}{f\left(x^{\prime}\right)},
$$


The WWO algorithm mainly simulates the dynamic motion of water wave and obtains the global optimal solution in the search space. The process is from the deep water area to shallow water area. In deep water area, waves have lower fitness values, longer wavelengths, and lower wave heights; in shallow water area, waves have higher fitness values, shorter wavelengths, and higher wave heights. The propagation, breaking, and refraction provide a good search mechanism for water wave optimization algorithm. Local search increases the depth of the algorithm and global search increases the breadth of the algorithm. The propagation can make the water wave with a higher fitness value perform local search in a small range, and the water wave with a lower fitness value perform global search in a large range. The breaking turns the optimal water waves into a series of isolated waves, which enhances the dense search near the global optimal solution and improves the calculation accuracy of the algorithm. The refraction can remove the energy-depleted waves, which effectively avoids search stagnation and increases population diversity, generate better waves will replace the poor waves, and it can accelerate the convergence speed of the algorithm. The combination of three operations effectively balances the global search and local search of the water wave optimization algorithm. The solution procedure is given in Algorithm 1.

Algorithm 1 The solution procedure of the WWO algorithm

Input: Define objective function;

Output: Global optimal solution $x^{*}$;

Step 1. Randomly initialize a water wave population $P$ with $n$ waves (solutions) that contains parameters $\alpha, \beta$, and $\lambda$;

Step 2. while stop the termination criterion is not satisfied do

Step 3. for each water wave $x \in P$ do

Step 3.1. propagate $x$ to a new $x^{\prime}$ by using Eq. (6);

Step 3.2. if $f\left(x^{\prime}\right)>f(x)$ then

Step 3.2.1. if $f\left(x^{\prime}\right)>f\left(x^{*}\right.$ then

Break $x^{\prime}$ by using Eq. (8);

Update $x^{*}$ with $x^{\prime}$;

Step 3.2.2. Replace $x$ with $x^{\prime}$;
Step 3.3. else Decrease $x . h$ by one;

$$
\text { if } x . h=0 \text { then }
$$

Refract $x$ to a new $x^{\prime}$ by using Eq. (9) and (10);

Step 4. Update the wavelengths by using Eq. (7);

Step 5. return $x^{*}$.

\section{PID Controller Based on the WWO Algorithm}

The PID controller is a linear controller that is used to improve the dynamic response to correct the steady state error. PID controller is applied to the AVR system to improve the terminal voltage step response. The PID controller transfer function is given as:

$$
\frac{\Delta V_{e}(s)}{U(s)}=K_{p}+\frac{K_{i}}{s}+K_{d} s,
$$

where $K_{p}, K_{i}, K_{d}$ represent the proportional gain, the integral gain and the differential gain, respectively.

In order to gain better performance control, the optimal parameters of PID controller are obtained by WWO algorithm. Transfer function model of an AVR system with WWO-PID controllers is given in Figure 2. The maximum overshoot, rise time, steady sate error are used as a typical time domain analysis index. In this paper, the Integral Time multiplied by the Absolute Error (ITAE) is regarded as a performance indicator in the control design. ITAE is an objective function that contains a time value. ITAE can be given as:

$$
\text { ITAE }=\int_{0}^{t} t\left|V_{t}-V_{r e f}\right| d t,
$$

where $t$ is the simulation time, $V_{t}$ is the terminal voltage and $V_{\text {ref }}$ is the reference voltage.

The WWO algorithm has a strong global search ability, which can find the optimal control variables $\left(K_{p}\right.$, $K_{i}, K_{d}$ ) and the global optimal solution. The lower and upper bounds of the three controller parameters are given in Table 2. The correspondence between the problem space and WWO algorithm space is shown in Table 3. 


\section{Figure 2}

Transfer function model of an AVR system with WWO-PID controllers

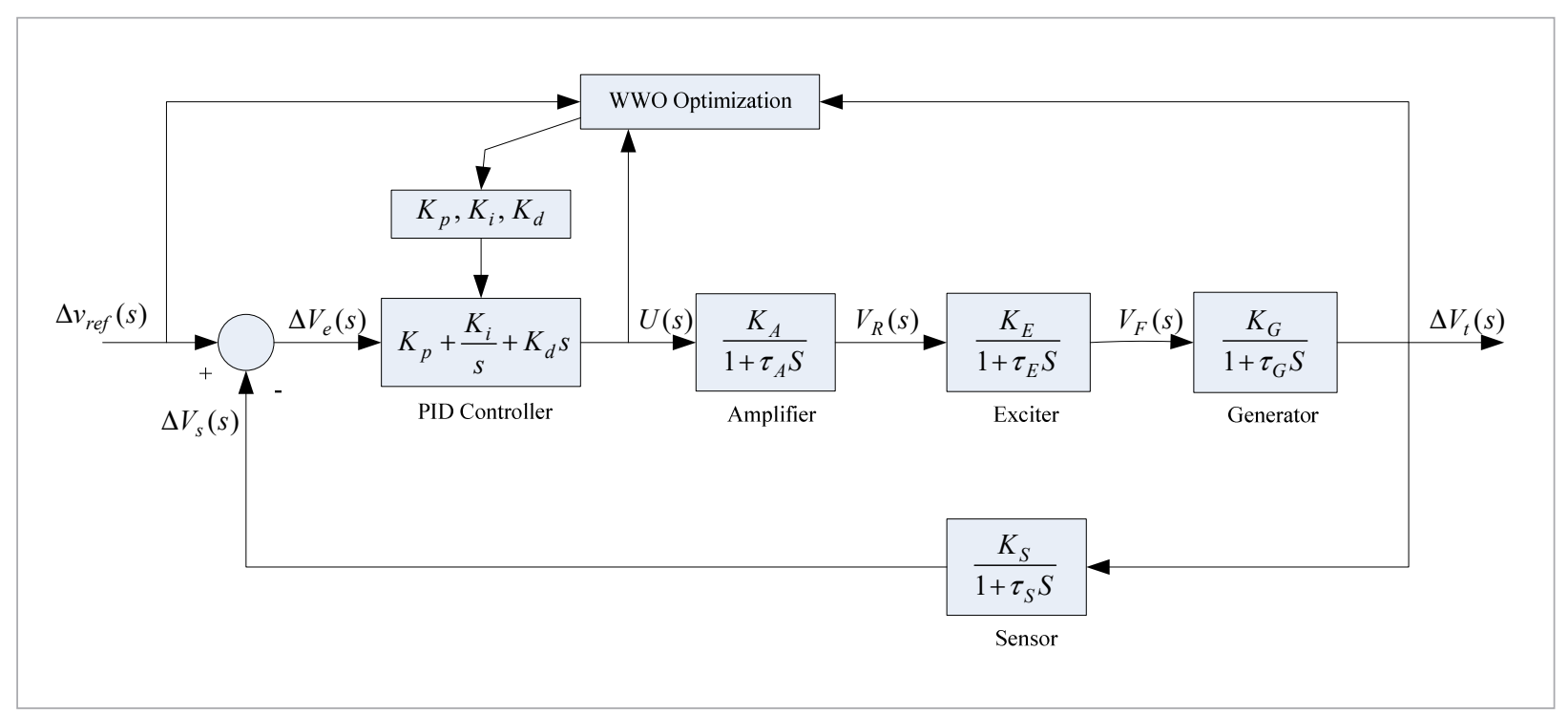

Table 2

Range of three controller parameters

\begin{tabular}{c|c|c}
\hline Controller parameters & Min. value & Max. value \\
\hline$K_{p}$ & 0 & 1.5 \\
\hline$K_{i}$ & 0 & 1.0 \\
\hline$K_{d}$ & 0 & 1.0 \\
\hline
\end{tabular}

\section{Table 3}

Correspondence between ORPD and WWO

\begin{tabular}{l|l}
\multicolumn{1}{c|}{ The problem space } & \multicolumn{1}{c}{ The Wwo algorithm space } \\
\hline $\begin{array}{l}\text { A collection contains all the optimization schemes } \\
\left(x_{1}, x_{2}, \ldots, x_{k}\right) \text { to optimize the PID controller parameters in AVR } \\
\text { system }\end{array}$ & A water wave population $P$ with $\left(n_{1}, n_{2}, \ldots, n_{k}\right)$ waves \\
\hline $\begin{array}{l}\text { An optimal optimization scheme that obtains proportional } \\
\text { gain } K_{p}, \text { the integral gain } K_{i} \text { and the differential gain } K_{d} \cdot\end{array}$ & An optimal water wave \\
\hline The objective evaluation function ITAE & The fitness function of the WWO algorithm
\end{tabular}

The flowchart of parameters tuning of PID using WWO algorithm is depicted in Figure 3 and the solution procedure in AVR system is given in Algorithm 2. 
Algorithm 2 The solution procedure in AVR system

Step 1. Determine the parameters of the AVR system, proportional gain $K_{p}$, the integral gain $K_{i}$ and the differential gain $K_{d}$;

Step 2. Randomly initialize a population $P$ of $n$ waves (solutions), iter $=0$, the wave length reduction coefficient $\alpha$, the breaking coefficient $\beta$, the wave length $\lambda$, and the maximum number iter $_{\max }$,;

Step 3. Specify the lower and upper bounds on the three controller parameters, for each water wave, apply PID controller with gains specified by that water wave to AVR system and run the whole system steps, calculate the fitness value of each water wave by Eq. (12);

Step 4. Find out the optimal water wave

While stop criterion is not satisfied do

Step 5. for each wave $x \in P$ do

Step 5.1. Propagate water wave $x$ to a new wave $x^{\prime}$ by Eq. (6).

Step 5.2. if $f\left(x^{\prime}\right)>f(x)$ then

Step 5.2.1. if $f\left(x^{\prime}\right)>f\left(x^{*}\right)$, then break water wave $x^{\prime}$ by Eq. (8), the current optimal wave $x^{*}$ will be replaced by water wave $x$.

Step 5.2.2. The original water wave $x$ is replaced by the new water wave $x$ ' in the population.

Step 5.3. else, wave height $h$ of wave $x$ is reduced by one, which indicates energy consumption. If $x . h=0$, then the wave $x$ will perform refraction operation according to Eq.(9) and (10).

Step 5.4. Update the wavelength of all water waves according to Eq. (7).

Step 6. calculate the fitness function of each water wave by Eq. (12);

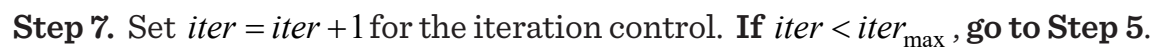

Step 8. Output the best solution and an optimal controller parameters.

\section{Experiment Results and Analysis}

This section mainly contains two important contents: Subsection 5.1 simply introduces the experimental setup; Subsection 5.2 minutely expounds the WWO algorithm to optimize PID controller parameters in the AVR system.

\subsection{Experimental Setup}

All of the algorithms were programmed in MATLAB R2012a, and numerical experiment was set up on AMD Athlont (tm) II*4640 processor and 4 GB memory.

\subsection{WWO Algorithm to Optimize PID Controller Parameters}

The WWO algorithm is applied to optimize the PID controller for an AVR system, which finds a set of op- timal gains that minimize the value of the objective function. In order to better prove the superiority of the WWO algorithm, we compare the WWO [20] with other algorithms which include BA [23], CSA [1], FPA [24], PSO [7] and SCA [12]. The result indicates the WWO algorithm obtains better performance. The important parameters of all algorithms are given in Table 4.

In this work, the WWO algorithm obtains the optimal gains by using the population size of 50, 20 iterations, and 20 runs independently. The "Best" is the optimal fitness value, "Worst" is worst fitness value, "Mean" is mean fitness value and "Std." is standard deviation. The optimization result is given in Table 5. The ranking is based on standard deviation. The terminal voltage curves of the AVR system for different algorithms are given in Figure 3. 
Table 4

Important parameters of six algorithms

\begin{tabular}{c|l}
\hline Algorithms & \multicolumn{1}{c}{ Parameter values } \\
\hline BA & $A=0.25, r=0.5, f \in[0,2]$, the population size is 50. \\
\hline CSA & $r \in[0,1], f=2, a A P=0.1$, the population size is 50. \\
\hline FPA & $\rho=0.8$, the population size is 50. \\
\hline PSO & $c_{1}=1.4962, c_{2}=1.4962, \omega=0.7298$, the population size is 50. \\
\hline SCA & $r_{2} \in[0,2 \pi], a=2, r_{4} \in[0,1]$, the population size is 50. \\
\hline WWO & $\lambda=0.5, h_{\max }=6, \alpha=1.0026, \beta \in[0.01,0.25], k_{\max }=\min (12, \mathrm{D} / 2)$, the population size is 50. \\
\hline
\end{tabular}

Table 5

Parameters used in the six algorithms

\begin{tabular}{l|c|c|c|c|c|c|cr}
\hline \multicolumn{1}{c|}{ Control variables } & BA & CSA & FPA & PSO & SCA & WWO \\
\hline$K_{p}$ & 1.2397 & 1.2963 & 1.4117 & 1.2257 & 1.2517 & 1.2299 \\
\hline$K_{i}$ & 0.8581 & 0.6275 & 0.9743 & 0.8437 & 0.8609 & 0.8474 \\
\hline$K_{d}$ & 0.3946 & 0.2341 & 0.4561 & 0.3938 & 0.3986 & 0.3921 \\
\hline Peak amplitude(V) & 0.871 & 1.152 & 1.164 & 1.136 & 1.121 & 1.128 \\
\hline Settling time (sec) & 1.630 & 1.425 & 1.920 & 0.900 & 0.910 & 0.900 \\
\hline Rise time (sec) & 0.325 & 0.245 & 0.200 & 0.205 & 0.210 & 0.190 \\
\hline Best & 0.0328 & 0.0329 & 0.0330 & 0.0328 & 0.0328 & 0.0327 \\
\hline Worst & 0.0395 & 0.0372 & 0.0380 & 0.0409 & 0.0382 & 0.0361 \\
\hline Mean & 0.0339 & 0.0344 & 0.0341 & 0.0346 & 0.0344 & 0.0337 \\
\hline Std & 0.0019 & 0.0012 & 0.0013 & 0.0021 & 0.0015 & $7.8242 \mathrm{e}-04$ \\
\hline Rank & 5 & 2 & 3 & 6 & 4 & 1
\end{tabular}

Figure 3

Terminal voltage curves of the AVR system for different algorithms

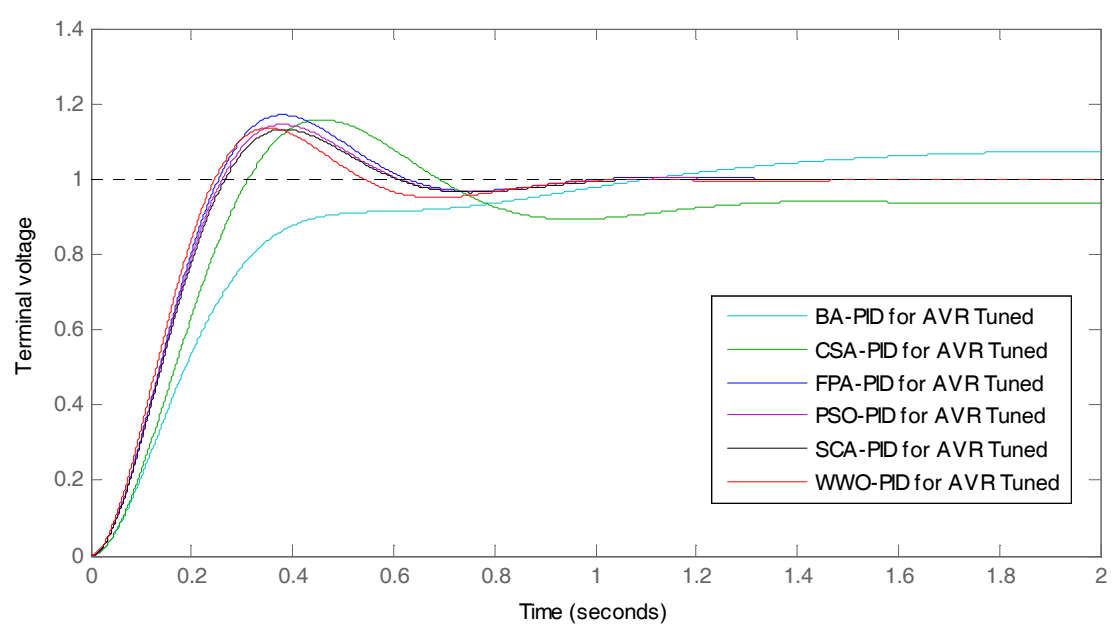


As can be seen in Table 5, the WWO algorithm has a strong global search and local search capability, which can find the optimal parameters of PID controller and global solution. The WWO algorithm could accelerate the convergence speed and improve the calculation accuracy. The robustness of PID controller is verified by the WWO algorithm to improve the step transient response of the AVR system of a generator. Compared with other algorithms, the peak amplitude, settling time and rise time of the WWO algorithm are better. The WWO algorithm can find the optimal fitness value, worst fitness value, mean fitness value and standard deviation. This indicates that the WWO algorithm is mainly utilized to determine three optimal AVR system parameters. The controller system could obtain on optimal solution, which demonstrates the efficiency of the PID controller.

\section{Figure 4}

Evolution curves of fitness value

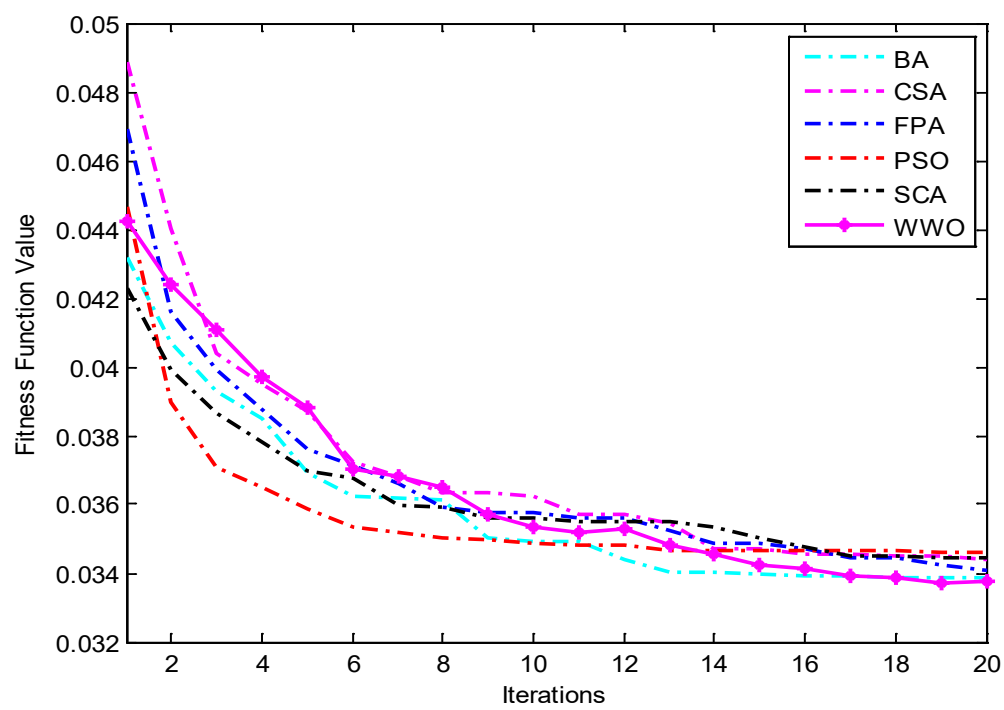

\section{Figure 5}

Anova test of global minimum

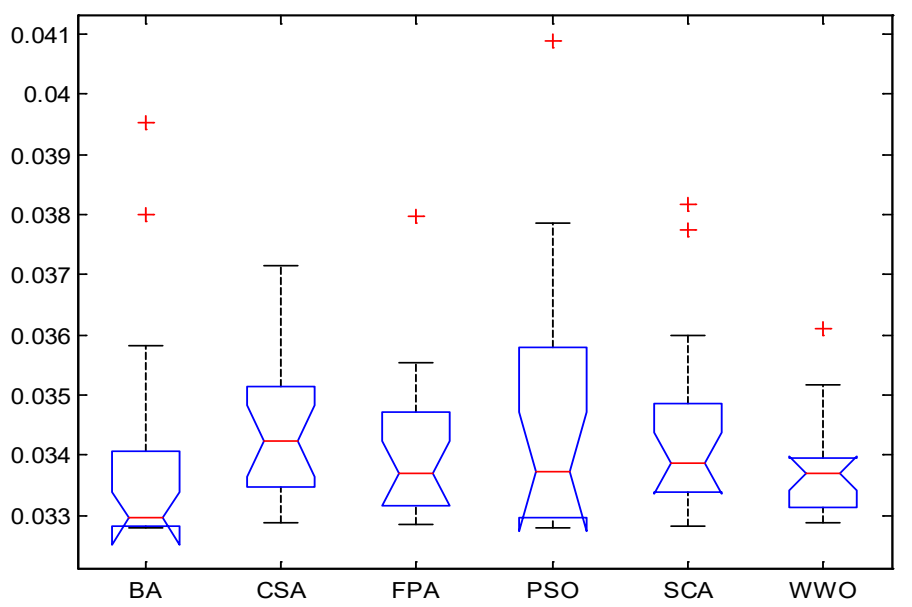


As can be seen in Figures 4-5, the WWO algorithm has a strong global search capability. Although the convergence speed of the WWO algorithm is not faster, the calculation accuracy of the WWO algorithm is the best among the six algorithms. The WWO algorithm is used to optimize the PID controller parameters in AVR system, which has a good application prospect. The WWO algorithm obtains the optimal gain parameters, so that it finds the optimal fitness value. This indicates that the WWO algorithm can better balance the exploration and the exploitation. Compared with other algorithms containing BA, CSA, FPA, PSO and SCA, the standard deviation of the WWO algorithm is much better, which indicates that the robustness and stability is stable. Generally speaking, the overall performance of the WWO algorithm is superior to that of other algorithms.

All the algorithms are applied to solve the PID controller parameter optimization, aiming to find the global optimal solution. Each algorithm runs independently 20 times, independent execution time is given in Table 6. Compared with other algorithms, the independent running time of the WWO algorithm is relatively small. The execution time of different algorithms can be seen in Figure 6.

Table 6

Execution time of different algorithms

\begin{tabular}{c|c|c|c|c|c|c|}
\hline Algorithm & BA & CSA & FPA & PSO & SCA & WWO \\
\hline Time execution (Seconds) & 63 & 60 & 56 & 124 & 69 & 59 \\
\hline
\end{tabular}

\section{Figure 6}

Execution time Vs different algorithms

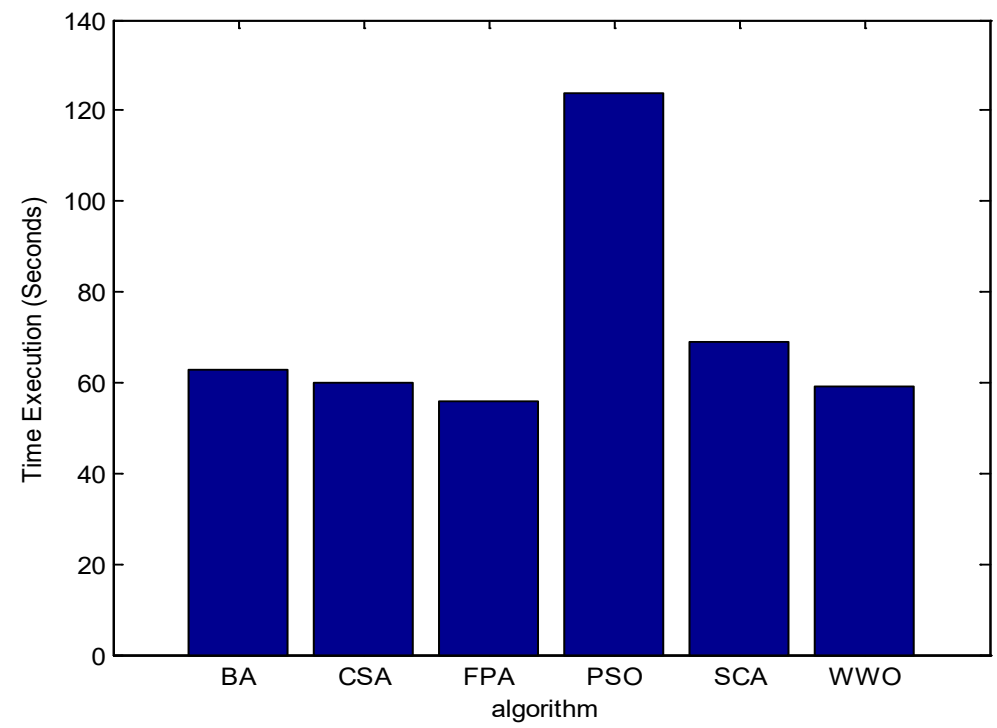

\section{Conclusion}

In this paper, the WWO algorithm is used to determine the parameters of the PID controller for an AVR system. The WWO algorithm contains propagation operation, breaking operation and refraction opera- tion, which has a strong global search ability and local search ability, so that it can obtain the optimal solution to the problem. Compared with other algorithms, such as BA, CSA, FPA, PSO and SCA, the WWO algo- 
rithm could not only find the optimal controller gains and the best fitness value, but also the standard deviation is the smallest. In order to expand the application area of the water wave optimization algorithm, future researches will be applied to the medical field, such as medical image segmentation, cardiac diagnosis, pharmacokinetics, and fault diagnosis.

\section{References}

1. Askarzadeh, A. A Novel Metaheuristic Method for Solving Constrained Engineering Optimization Problems: Crow Search Algorithm. Computers and Structures, 2016, 169, 1-12. https://doi.org/10.1016/j.compstruc.2016.03.001

2. Chatterjee, S., Mukherjee, V. PID Controller for Automatic Voltage Regulator Using Teaching-learning Based Optimization Technique. International Journal of Electrical Power and Energy Systems, 2016, 77, 418429. https://doi.org/10.1016/j.ijepes.2015.11.010

3. Gaing, Z. L. A particle Swarm Optimization Approach for Optimum Design of PID Controller in AVR System. IEEE Transactions on Energy Conversion, 2004, 19(2), 384-391. https://doi.org/10.1109/TEC.2003.821821

4. Gozde, H., M. C. Taplamacioglu. Comparative Performance Analysis of Artificial Bee Colony Algorithm for Automatic Voltage Regulator (AVR) System. Journal of the Franklin Institute, 2011, 348(8), 1927-1946. https:// doi.org/10.1016/j.jfranklin.2011.05.012

5. Hasanien, H. M. Design Optimization of PID Controller in Automatic Voltage Regulator System Using Taguchi Combined Denetic Algorithm Method. IEEE Systems Journal, 2013, 7(4), 825-831.https://doi.org/10.1109/ JSYST.2012.2219912

6. Hematabadi, A. A., Foroud, A. A. Optimizing the Multi-objective Bidding Strategy Using Min-Max Technique and Modified Water Wave Optimization Method. Neural Computing \& Applications, 2018, 6, 1-19. https:// doi.org/10.1007/s00521-018-3361-0

7. Kennedy, J., Eberhart, R. Particle Swarm Optimization. IEEE International Conference on Neural Networks, 1995, 4(8), 1942-1948. https://doi.org/10. 1109/ ICNN.1995.488968

8. Kilany, M., Hassanien, A. E., Badr, A. Accelerometer-Based Human Activity Classification Using Water Wave Optimization Approach. Computer Engineering Conference, 2016, 175-180. https://doi.org/10.1109.ICENC0.2015.7416344

\section{Acknowledgments}

This work was supported by the National Science Foundation of China under Grant Nos. 61563008, and Project of the Guangxi Natural Science Foundation under Grants No. 2016GXNSFAA380264. 2018 GXNSFAA138146.

9. Lakshminarasimman, L., Siva, M., Balamurugan, R. Water Wave Optimization Algorithm for Solving Multi-Area Economic Dispatch Problem. International Journal of Computer Applications, 2017, 167(5), 19-27. https://doi.org/10.5120/ijca2017914247

10. Lenin, K., Ravindhranath Reddy, B., Suryakalavathi, M. Hybridization of Firefly and Water Wave Algorithm for Solving Reactive Power Problem. International Journal of Engineering Research in Africa, 2015, 21, 165-171. https:// doi.org/10.4028/www.scientific.net/JERA.21.165

11. Li, C., Li, H., Kou, P. Piecewise Function Based Gravitational Search Algorithm and Its Application on Parameter Identification of AVR System. Neurocomputing, 2014, 124(2), 139-148. https://doi.org/10.1016/j.neucom.2013.07.018

12. Mirjalili, S. SCA: A Sine Cosine Algorithm for Solving Optimization Problems. Knowledge-Based Systems, 2016, 96,120-133. https://doi.org/10.1016/j.knosys.2015.12.022

13. Mitsukura, Y., Yamamoto, T., Kaneda, M. A Design of Self-Tuning PID Controllers Using a Genetic Algorithm. American Control Conference, 1999, 36(1), 13611365. https://doi.org/10.1109/ACC.1999.783590

14. Odili, J. B., Mohmad, M. K., Noraziah, A. Parameters-Tuning of PID Controller for Automatic Voltage Regulators Using the African Buffalo Optimization: Plos One, 2017, 12(4), e0175901. https://doi.org/10.1371/journal. pone.0175901

15. Ota, T., Omatu, S. Tuning of the PID Control Gains by GA. Emerging Technologies and Factory Automation, 1996, 1(1), 272-274. https://doi.org/10.1109/ ETFA.1996.573305

16. Panda, S., Sahu, B. K., Mohanty, P. K. Design and Performance Analysis of PID Controller for an Automatic Voltage Regulator System Using Simplified Particle Swarm Optimization. Journal of the Franklin Institute, 2012, 349(8), 2609-2625. https://doi.org/10.1016/j. jfranklin.2012.06.008 
17. Siva, M., Balamurugan, R., Lakshminarasimman, L. Water Wave Optimization Algorithm for Solving Economic Dispatch Problems with Generator Constraints. International Journal of Intelligent Engineering \& Systems, 2016, 9(4), 31-40. https://doi.org/10.22266/ ijies2016.1231.04

18. Siva, M., Balamurugan, R., Lakshminarasimman, L. Water Wave Optimization Algorithm for Solving Combined Economic And Emission Dispatch Problem. Journal of Engineering \& Applied Sciences, 2016, 12(6), 1730-1741. https://www.researchgate./net/publication/316595942

19. Tang, Y., Zhao, L., Han, Z., Bi, X., Guang, X. Optimal Gray PID Controller Design for Automatic Voltage Regulator System via Imperialist Competitive Algorithm. International Journal of Machine Learning and Cybernetics, 2016, 7(2), 229-240. https://doi.org/10.1007/s13042015-0431-9

20. Wu, X., Zhou, Y., Lu, Y. Elite Opposition-Based Water Wave Optimization Algorithm for Global Optimization. Mathematical Problems in Engineering, 2017, 2017(11), 1-25. https://doi.org/10.1155/2017/3498363

21. Wu, X.-B., Liao, J., Wang, Z.-C. Water Wave Optimization for the Traveling Salesman Problem. International Conference on Intelligent Computing, 2015, 9225, 137146. https://doi.org/10.1007/978-3-3-319-22180-9_14

22. Wang, S., Xu, Y., Pen, Y. A Fast Image Match Method Based on Water Wave Optimization and Gray Relational Analysis. IEEE International Conference on Intelligent
Data Acquisition and Advanced Computing Systems: Technology and Applications, 2017, 771-776.https://doi. org/10.1109/IDAACS.2017.8095193

23. Yang, X., He, X. Bat Algorithm: Literature Review and Applications. International Journal of Bio-Inspired Computation, 2013, 5(3), 141-149. https://doi. org/10.1504/IJBIC.2013.055093

24. Yang, X. Flower Pollination Algorithm for Global Optimization. Unconventional Computation and Natural Computation. Springer Berlin Heidelberg, 2012, 7445(2), 242243. https://doi.org/10.1007/978-3-642-32894-7__27

25. Yun, X., Feng, X., Lyu, X. A Novel Water Wave Optimization Based Memetic Algorithm for Flow-Shop Scheduling. 2016 IEEE Congress on Evolutionary Computation (CEC), 2016, 1971-1976. https://doi.org/10.1109/ CEC.2016.77444029

26. Zhang, J., Zhou, Y., Luo, Q. An Improved Sine Cosine Water Wave Optimization Algorithm for Global Optimization. Journal of Intelligent \& Fuzzy Systems, 2018, 34(4), 2129-2141. https://doi.org/10.3233/JIFS-171001

27. Zhao, S., Li, Z., Yun, X., Wang, K., Lyu, X., Liu, B. IIR Filters Designing by Water Wave Optimization. IEEE International Conference on Control \& Automation, 2017, 347-352. https://doi.org/10.1109/ICCA.2017.8003085

28. Zheng, Y. Water Wave Optimization: A New Nature-Inspired Metaheuristic. Computers and Operations Research, 2015, 55, 1-11. https://doi.org/10.1016/j. cor.2014.10.008 